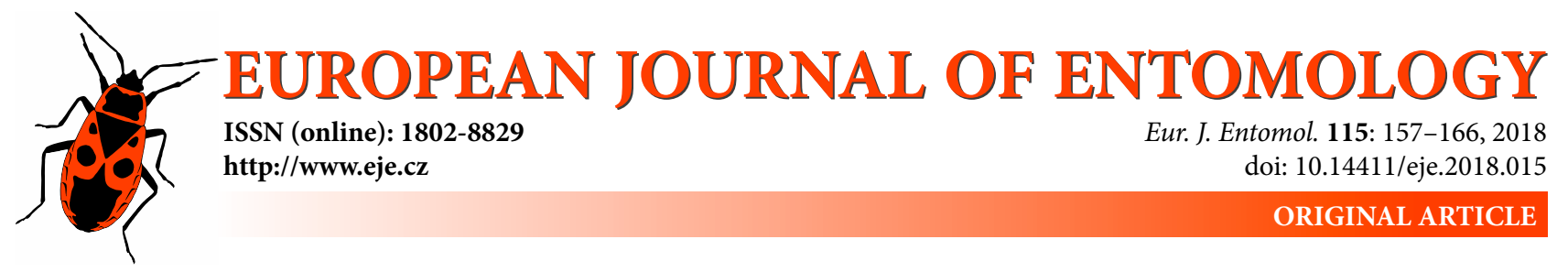

\title{
Molecular cloning and functional analyses of an adhesion molecule, neuroglian, in Mythimna separata (Lepidoptera: Noctuidae)
}

\author{
Kakeru YOKOI*, Yoshiaki KATO**, Masahiro SUZUKI and Ken MIURA \\ Applied Entomology, Graduate School of Bio-Agricultural Sciences, Nagoya University, Chikusa, Nagoya 464-8601, Japan; \\ e-mails: yokoi123@affrc.go.jp, m \\ sssuzuki@gmail.com, k-miura@agr.nagoya-u.ac.jp
}

Key words. Lepidoptera, Noctuidae, Mythimna separata, neuroglian, hemocyte adhesion, encapsulation, in situ hybridization, real-time quantitative reverse transcript PCR, RNA interference

\begin{abstract}
Insect cellular immune reaction, which generally includes phagocytosis, encapsulation and nodule formation, is achieved by hemocytes circulating in insect haemolymph. The shift of hemocytes from the normal phase to the adhering phase is an important process in the cellular immune reaction, which includes the attachment of hemocytes to foreign surfaces or other hemocytes via adhesion factors. Neuroglian is one of the adhering factors associated with encapsulation in Manduca sexta and Drosophila melanogaster. Here we studied the localization of neuroglian (MsNrg) in Mythimna separata and its functional role in the cellular immune reaction. The distribution of $\mathrm{MsNrg}$ mRNA between hemocyte populations was determined using real-time quantitative reverse transcription PCR and in situ hybridization, which revealed that MsNrg was highly expressed in adhering hemocytes, especially in plasmatocytes. Unexpectedly, the transcript was observed as well in non-adhering hemocytes, implying neuroglian has a function in non-adhering hemocytes. Moreover, we showed that the amount of MsNrg mRNA was not changed by injections of either biotic or abiotic non-selves. Fewer latex beads were fully encapsulated by hemocytes in larvae treated with $\mathrm{MsNrg}$ double-stranded RNA than in control larvae, but their ability to achieve phagocytosis and nodule formation remained unchanged by the $\mathrm{MsNrg}$ knockdown. These results indicate that the function of neuroglian in the cellular immune reaction is conserved in $D$. melanogaster and lepidopteran species, and neuroglian in non-adhering hemocytes could possess unidentified function.
\end{abstract}

\section{INTRODUCTION}

Insects do not have adaptive immune systems, which are based on genomic rearrangement of immune genes. Instead, they have quite robust innate immune systems, on which they solely rely. The latter immune system, like the former, consists of humoral and cellular defenses. The humoral immune defense of insects is composed of several distinct levels of reactions. For example, the invasion of pathogens induces the production of several antimicrobial peptides and reactive oxygen/nitrogen species (Lemaitre \& Hoffmann, 2007). Molecular mechanisms and pathways of insect humoral immune defense have been studied intensively in a model insect, Drosophila melanogaster Meigen (Diptera: Drosophidae). Cellular immune defense is mediated mainly by hemocytes as shown in vertebrate systems (Lavine \& Strand, 2002). These hemocytes can be divided into several distinct types according to their shapes and functions. In larval stages of typical lepido- pteran insects, five types of hemocytes are usually found: plasmatocytes, granular cells, spherule cells, oenocytoids and prohemocytes. Plasmatocytes and granular cells are adhering hemocytes, which are considered to play a central role in cellular immune defense in Lepidoptera (Lavine \& Strand, 2002). Insect cellular immune defense mainly consists of phagocytosis, encapsulation and nodulation. During phagocytosis, hemocytes engulf small abiotic (such as small chromatography beads) or biotic targets (microbes and apoptotic bodies) (Hernandez et al., 1999; Da Silva et al., 2000). In Lepidoptera, phagocytosis is carried out mainly by granular cells and plasmatocytes. Encapsulation is another cellular immune defense during which adhering hemocytes attach to non-self targets, which are too large to engulf (parasitoids, nematodes and large beads), and form multiple cell layers around them to physically isolate and kill the target (Lavine \& Strand, 2002). Nodulation is initiated by small but numerous targets. In this case, hemo-

\footnotetext{
* Present address: Insect Genome Research and Engineering Unit, Division of Applied Genetics Institute of Agrobiological Sciences, National Agriculture and Food Research Organization (NARO), 1-2 Owashi, Tsukuba, Ibaraki, 305-8634, Japan; e-mail: yokoi123@affrc.go.jp

** Present address: BASF Japan Ltd., Roppongi Hills Tower 21F, 6-10-1 Roppongi, Minato-ku, Tokyo 106-6121, Japan; e-mail: Katoooooo@yahoo.co.jp
} 
cytes attach to aggregated bacteria, which are eventually surrounded and killed by multiple cell layers, as is seen in the process of encapsulation (Lavine \& Strand, 2002; Marmaras \& Lampropoulou, 2009).

These cellular reactions are thought to be controlled by several cell adhesion molecules on the surfaces of hemocytes; these molecules are responsible for the shift between the non-adhesion and adhesion status of hemocyte (Nardi et al., 2005). Therefore, it is quite important to investigate cell adhesion molecules for understanding these cellular immune defense mechanisms. Although there is a large amount of information on the molecular mechanisms of humoral immune defense, the mechanisms of cellular immune defense, such as molecular basis for hemocyte aggregation and intercellular signalling, recognition of biotic or abiotic non-selves and regulation of hemocyte movements, are far from being described in detail.

We have been interested in the host regulation mechanisms exerted by parasitoid wasps against their host larvae in which parasitoid-derived factors are known to regulate the lepidopteran host's immune defenses (Suzuki et al., 2008). The target molecules of these parasitoid-derived factors could include adhesion molecules on the surfaces of the host's hemocytes. One of these adhesion molecules in the immunoglobulin superfamily is neuroglian (Hortsch, 1996; Wiegand et al., 2000; Nardi et al., 2003, 2005). Neuroglian was first reported in D. melanogaster (Bieber et al., 1989), and subsequently described in a lepidopteran, the tobacco hornworm, Manduca sexta (Linnaeus) (Lepidoptera: Sphingidae) (Chen et al., 1997). Neuroglian is homologous to vertebrate neural adhesion molecule L1, and its expression was first shown in developing nerves of D. melanogaster (Bieber et al., 1989). Since then, main research interest in Drosophila neuroglian has been in the formation and growth of a synapse by using mutant fly lines and cultured cells (Hortsch et al., 1995; Hall \& Bieber, 1997; Siegenthaler et al., 2015). In the meanwhile, Nardi, Kanost and co-workers noticed the occurrence of neuroglian proteins on the surfaces of the hemocytes of the lepidopteran, $M$. sexta, and reported its distribution in hematopoetic organs and its functional involvement in encapsulation (Nardi, 1994; Nardi et al., 2003, 2006; Zhuang et al., 2007). After the series of publications, involvement of D. melanogaster neuroglian in encapsulating a parasitoids wasp's egg was shown (Williams, 2009).

To extend the study of neuroglian's involvement in cellular immune defense, we first determined full nucleotide sequences of the Mythimna separata (Walker) (Noctuidae: Lepidoptera) neuroglian ( $M s N r g$ ). Subsequently, we investigated the abundance and distribution of $M s N r g$ mRNA in different types of hemocytes using real-time quantitative reverse transcription PCR (qRT-PCR). As functional assay of the involvement of MsNrg in cell-mediated immune responses in phagocytosis, encapsulation and nodulation, we conducted knockdown assays using RNA interference (RNAi).

\section{MATERIALS AND METHODS}

\section{Insect rearing}

M. separata was reared as described in Suzuki \& Tanaka (2007) and Nakamatsu et al. (2006). Briefly M. separata were collected in cornfields in Kanoya, Kagoshima Prefecture, Japan. These insects were reared on an artificial diet (Insecta LFs, Nihon Nosan Co., Kanagawa Prefecture, Japan) under a long day photoregime $(16 \mathrm{~L}: 8 \mathrm{D})$ at $25 \pm 1^{\circ} \mathrm{C}$.

\section{RNA extraction and CDNA synthesis}

The sixth instar, day-0 (VI-0, hereafter abbreviated in this manner) larvae were anesthetized with carbon dioxide gas. Their hemolymph was collected in ice-cold phosphate-buffered saline (PBS), and the total hemocytes were isolated by centrifugation at 2,200 rpm for $10 \mathrm{~min}$ at $4^{\circ} \mathrm{C}$. Total RNA was extracted from these samples using TRIZOL reagent (Invitrogen, Carlsbad, CA, USA) according to the manufacturer's instructions. The first strand cDNA synthesis was done by either PrimeScript reverse transcriptase (TAKARA, Kusatsu, Shiga, Japan) or Thermoscript reverse transcriptase (Thermo Fisher Science, Waltham, MA, USA), primed with three distinct primers, random hexamer, oligo-(dT) 20 and a tagged oligo-(dT) primer. The sequence of the tagged oligo-(dT) primer used for 3' RACE is: 5'-CTACAGTCTGCTCACAGCATAGTATTTTTTTTTTTTTTTTTTTTTTTTTVN-3'. For 5' RACE, an RNA oligo (5'-AAGCAGUGGUAUCAACGCAGAGUGGG-3') was included in the reverse transcription reaction according to the instruction of a SMART RACE cDNA amplification kit (Clontech, Mountain View, CA, USA).

\section{Homologous RT-PCR methods}

A pair of degenerate primers was designed based on the highly conserved regions of neuroglian proteins from four species of insects, D. melanogaster, Bombyx mori Linnaeus (Lepidoptera: Bombycidae), M. sexta and Apis mellifera Linnaeus (Hymenoptera: Apidae), 5'-ATGACKYTNGAYCCIGARGGIAA-3' for a forward primer and 5'-CAKCCRTARTTICCIGTRTCYTT-3' for a reverse primer. PCR following RT-PCR was performed using the oligo-(dT) 20 -primed first strand cDNA as described above under the following conditions: the reaction mixture was first kept at $94^{\circ} \mathrm{C}$ for $2 \mathrm{~min}$, followed by 35 cycles of denaturation at $94^{\circ} \mathrm{C}$ for $30 \mathrm{~s}$, annealing at $45^{\circ} \mathrm{C}$ for $1 \mathrm{~min}$ and extension at $72^{\circ} \mathrm{C}$ for $1 \mathrm{~min}$. A random hexamer-primed first strand cDNA preparation was also utilized to amplify a different region, closer to the 3' end of the putative neuroglian cDNA. In this case, reverse transcription was carried out using a thermostable enzyme, Thermoscript reverse transcsriptase at $65^{\circ} \mathrm{C}$, following $10 \mathrm{~min}$ incubation at $25^{\circ} \mathrm{C}$. RT-PCR was then performed with another pair of degenerate primers: 5'-GTNYTNACIGGITAYAARATITAYTA-3' as the forward primer and 5'-TCKCKRTCRTGIACRTCRTAITT-3' as the reverse primer. The thermal cycling conditions were as follows: $94^{\circ} \mathrm{C}$ for $2 \mathrm{~min}$, followed by 43 cycles of denaturation at $94^{\circ} \mathrm{C}$ for $30 \mathrm{~s}$, annealing at $50^{\circ} \mathrm{C}$ for $1 \mathrm{~min}$ and extension at $72^{\circ} \mathrm{C}$ for $1 \mathrm{~min}$. Respective PCR products were fractionated using agarose gel electrophoresis, and the cDNAs with expected sizes were recovered using a QIAquick Gel Extraction kit (QIAGEN, Hilden, North Rhine-Westphalia, Germany). The cDNA fragments were sub cloned into either pBluescript II SK+ (Stratagene, La Jolla, CA, USA) or pCR2.1-TOPO (Invitrogen). Several independent cDNA clones were sequenced using an ABI Prism Dye Terminator Cycle Sequencing kit and a DNA Sequencer (Model 3130, Applied Biosystems, Waltham, MA, USA). 


\section{Acquisition of neuroglian full nucleotide sequence by PCR-based methods}

The two partial sequences obtained from different regions of $\mathrm{MsNrg}$ using homologous RT-PCR procedures were used to design gene-specific primers (forward primer: 5'-GGCGAGTCAGCTGAGATCAAG-3', reverse primer: 5'-CTCCTTAGCTGGTAAAGTACTC-3') to clone the unidentified region between the two sequences. The RT-PCR products were separated on an agarose gel, and the cDNA with the expected size was recovered. The cDNA fragment was sequenced directly by primer walking.

Amplifying the MsNrg cDNA in the vicinity of its putative 3' end after a conventional reverse transcription reaction presented a technical challenge. Therefore, for 3' RACE, Thermoscript reverse transcsriptase was used for reverse transcription at a higher temperature. The reverse transcription reaction was primed with the tagged anchor primer at $42^{\circ} \mathrm{C}$ for $7 \mathrm{~min}$, and this was followed by incubation at $65^{\circ} \mathrm{C}$ for $60 \mathrm{~min}$. This cDNA template was used for the following 3' RACE with a gene-specific primer (5'-TACCATTGACGGCCCCATAGTGAA-3') and a 3' anchor primer (5'-CTACAGTCTGCTCACAGCATAGTA-3') under the following conditions: $94^{\circ} \mathrm{C}$ for $2 \mathrm{~min}$, followed by 35 cycles of denaturation at $94^{\circ} \mathrm{C}$ for $30 \mathrm{~s}$, annealing at $68^{\circ} \mathrm{C}$ for $30 \mathrm{~s}$ and extension at $72^{\circ} \mathrm{C}$ for $1 \mathrm{~min} 20 \mathrm{~s}$. The PCR products were fractionated on an agarose gel, and the cDNA fragments corresponding to the expected sizes $(600-800 \mathrm{bp})$ were recovered. The secondary PCR was done using these cDNA fractions with the 3' anchor primer and a nested gene specific primer (5'-CATAGTGAAGCCGGACGAGGACA-3'). The thermal cycles used were: $94^{\circ} \mathrm{C}$ for $2 \mathrm{~min}$, followed by 45 cycles of denaturation at $94^{\circ} \mathrm{C}$ for $30 \mathrm{~s}$, annealing at $68^{\circ} \mathrm{C}$ for $30 \mathrm{~s}$ and extension at $72^{\circ} \mathrm{C}$ for $1 \mathrm{~min}$. The secondary PCR products were separated by agarose gel electrophoresis, and analyzed by Southern blotting. The $660 \mathrm{bp}$ cDNA fragment (see Fig. 1) was used as a probe. A Gene Image Detection Kit (GE Healthcare UK Ltd, Amersham Place, England) was used for probe labelling and detection. The cDNA zone exhibiting a positive signal was recovered and sub cloned. Then, the positive colonies were identified by hybridization using the probe mentioned above, the plasmids were prepared, and their cDNA inserts were sequenced.

5' RACE was done by using a 5' anchor primer (5'-AAGCAGTGGTATCAACGCAGAGT-3') and a gene specific primer (5'-GTAGTCGAACGGTTTGCCGTTC-3') designed from the 670 bp region (see Fig. 1) under the following conditions: $94^{\circ} \mathrm{C}$ for $2 \mathrm{~min}$, followed by 35 cycles of denaturation at $94^{\circ} \mathrm{C}$ for $30 \mathrm{~s}$, annealing at $65^{\circ} \mathrm{C}$ for $30 \mathrm{~s}$, and extension at $72^{\circ} \mathrm{C}$ for $1 \mathrm{~min}$. For preparation of the template, see "RNA extraction and cDNA synthesis" section in "Material and methods".

The PCR products were fractionated on an agarose gel, and the cDNA band with the expected size was recovered and sequenced directly.

\section{Sequence analyses}

The editing and analyses of nucleotide and amino acid sequences were performed using GENETYX software (GENETYX corporation, Tokyo, Japan). Homology search and multiple alignment were performed using CLUSTAL W and BLAST programmes on the DNA Data Bank of Japan website (http://clustalw. ddbj.nig.ac.jp/top-j.html). Accession numbers of $\mathrm{M}$. sexta $\mathrm{Nrg}$ and D. melanogaster Nrg are U50719.1 and NM_001169234, respectively. Domain analysis was done using NCBI Conserved Domain Search programme on the National Center for Biotechnology Information website (Marchler-Bauer et al., 2017).

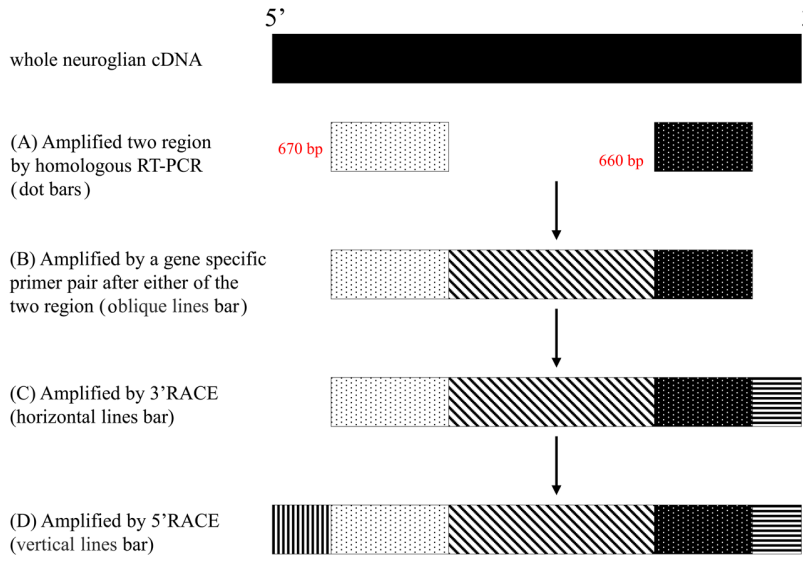

Fig. 1. Schematic drawing showing steps used in obtaining the full-length sequence. The top grey bar indicates the complete neuroglian cDNA. (A) Open and filled bars indicate the two regions obtained using homologous RT-PCR. Expected sizes for respective cDNA fragments are also shown. (B) The region between the two cDNA fragments (A) was amplified using conventional PCR methods and sequenced directly (diagonally hatched bar). (C) A gene specific primer was designed based on the 670 bp region (open bar) and used for the 5' RACE (vertically hatched bar). (D) Design of gene specific primers for 3' RACE and nested PCR was based on the sequence in the cDNA fragment indicated by the filled zone. This $660 \mathrm{bp}$ cDNA fragment was also used as a probe for Southern blotting. The region determined by the 3' RACE is indicated as a horizontally hatched bar. For the amplification of the $660 \mathrm{bp}$ fragment and the extreme 3' region, a thermostable reverse transcriptase was utilized. For details, see "Homologous RT-PCR methods" section of "Material and Methods".

\section{RNA interference}

Double-stranded RNA (dsRNA) was prepared as follows. First, T7 RNA polymerase promoter sequences were introduced into both 5' ends of the neuroglian double strand cDNA fragment (Fig. 2, DNA sequences are underlined) using PCR with the following primer pair: 5'-taatacgactcactatagggACGGGTATCGCGTCTACATT-3'; 5'-taatacgactcactatagggTTGGTGATGACGATGGTGTT-3'. The T7 RNA polymerase promoter sequences are in lower case. The $\mathrm{T} 7$ promoter-tagged cDNA was purified using a QIAquick PCR Purification kit (QIAGEN) and used as a template for dsRNA synthesis using a MEGAscript RNAi kit (Ambion, Carlsbad, CA, USA). For negative control, a dsRNA fragment possessing a partial enhanced green fluorescent protein (EGFP) sequence was also prepared in the same fashion by using the pEGFP-N1 plasmid (Clontech).

\section{qRT-PCR}

mRNA amounts were estimated using qRT-PCR. Hemolymph from four to six larvae was collected in Sf900 III media (Invitrogen) and was seeded in a 50-ml culture bottle. The hemocytes were allowed to attach the bottle for $30 \mathrm{~min}$, and non-adhering hemocytes were detached by tapping the bottle while leaving adhering ones attached. Total RNA was extracted separately from either the adhering or non-adhering hemocyte preparations. Total RNA was also extracted from the granular cell monolayer and plasmatocyte-enriched monolayer. Isolation of granular cells and plasmatocytes was done according to Suzuki et al. (2009). Reverse transcription of $1 \mu \mathrm{g}$ each of total RNA was primed by random hexamers. Each real-time RT-PCR reaction mixture ( 12.5 $\mu \mathrm{l})$ included $0.5 \mu \mathrm{l}$ of $1 \mathrm{st}$ strand cDNA, and the real-time detection and analyses were done based on SYBR green dye chemistry using a SYBR Premix Ex Taq Perfect Real Time kit (TAKARA). The thermal cycling conditions used for $M s N r g$ analysis were: 

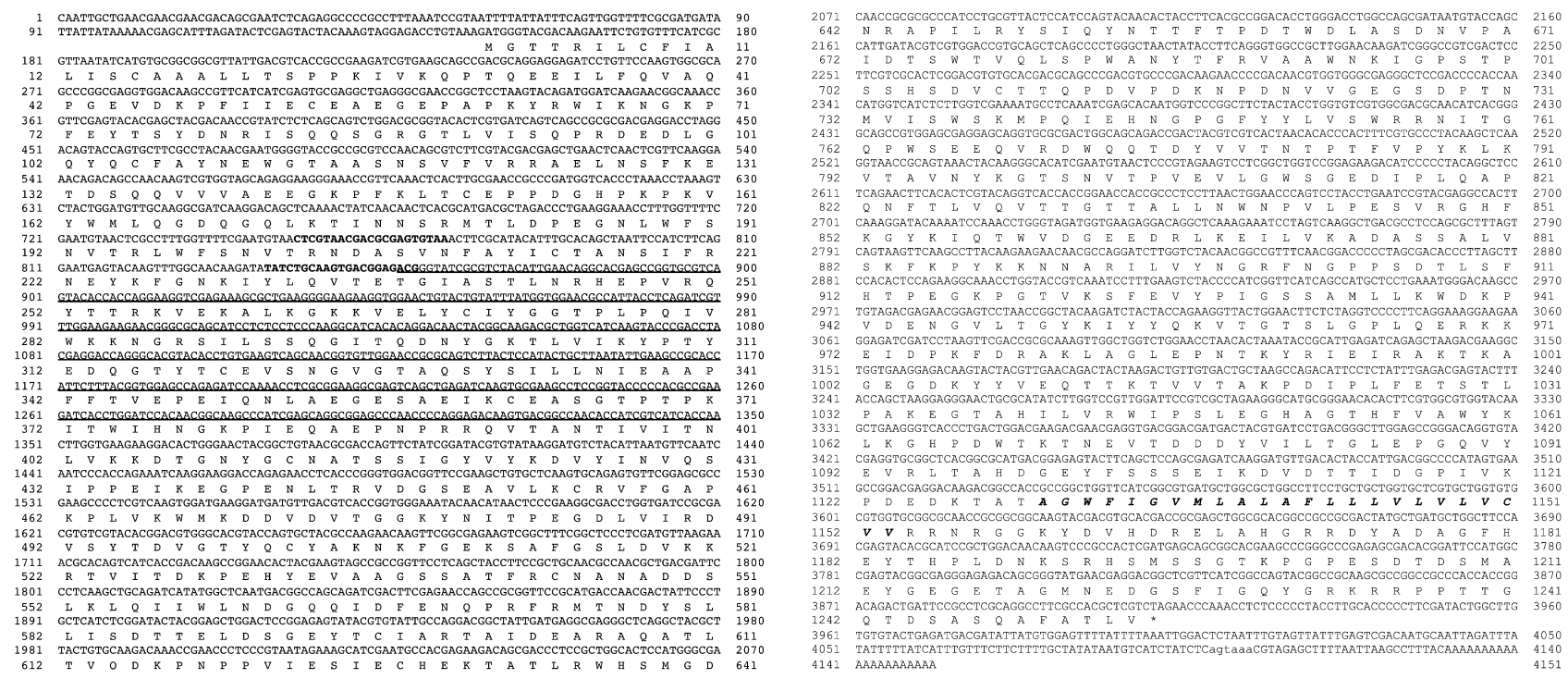

Fig. 2. Full nucleotide (upper) and predicted amino acid (lower) sequences of $M s N r g$ cDNA. Nucleotides are numbered from the first base at the 5 ' end. Amino acids are numbered from the initiating methionine residue. The polyadenylation signal atgaaa is designated by lower case letters. Amino acid residues, which may form a transmembrane domain, are in bold italics. The cDNA sequence used to synthesize MsNrg double strand RNA is underlined. The position of primer pair used for qRT-PCR is indicated by bold case letters.

$95^{\circ} \mathrm{C}$ for $10 \mathrm{~s}$, then 40 cycles of $95^{\circ} \mathrm{C}$ for $5 \mathrm{~s}, 60^{\circ} \mathrm{C}$ for $30 \mathrm{~s}$; this was followed by dissociation analysis composed of $95^{\circ} \mathrm{C}$ for 15 $\mathrm{s}, 60^{\circ} \mathrm{C}$ for $30 \mathrm{~s}$, then a shallow thermal ramp to $95^{\circ} \mathrm{C}$. The following primer pair was used: forward primer, 5'-CTCGTAACGACGCGAGTGTAA-3'; reverse primer, 5'-CGTCTCCGTCACTTGCAGATA-3'. The other primer pair for amplifying the house keeping gene [ribosomal protein L32 (RPL32)] was used under the same conditions. Each primer sequence was: 5'-GGTTACGGTTCAAACAAGAAGAC-3' for forward primer; 5'-TCCTGTTCTGCATCATCAGGAT-3' for reverse primer. Relative quantification for each mRNA was done based on a threshold cycle number determined by the second derivative of each primary amplification curve. The obtained mRNA values were normalized according to amount of RPL32 expressions or number of cells in the respective preparations.

\section{In situ hybridization}

In situ hybridization was done as described in Kato et al. (2014). The sequences of $M s N r g$ antisense and $M s N r g$ sense probes are 5'-CGTGGGGGTACCGGAGGCTTCGCACTTGATCTCAGCTGACTCGCCTTCCGCGAGG-3 and 5'CCTCGCGGAAGGCGAGTCAGCTGAGATCAAGTGCGAAGCCTCCGGTACCCCCACG-3, respectively.

\section{Phagocytosis assay}

The V-1 larvae were injected with $1 \mu \mathrm{g}$ of either $M s N r g$ or $E G F P$ dsRNA. Two days later, these larvae (ecdysed to VI-0 larvae) were injected with $5 \times 10^{6} \mathrm{DH} 5$ alpha Escherichia coli labelled with fluorescein isothiocyanate (FITC). Three hours later, the hemocytes were collected and resuspened in HEPES-buffered saline (HBS, $37.5 \mathrm{mM}$ Hepes buffer, $\mathrm{pH}$ 7.4, $100 \mathrm{mM} \mathrm{NaCl}, 2$ $\left.\mathrm{mM} \mathrm{CaCl}_{2}\right)$ containing $8 \%(\mathrm{v} / \mathrm{v})$ saturated phenylthiourea. These hemocyte suspensions were put on glass slides and left for 15 min. Then, they were washed three times with HBS, fixed in $70 \% \mathrm{EtOH}$ for $20 \mathrm{~min}$, washed three times with HBS again, and then mounted in HBS containing $40 \%$ glycerol. Five fields of view were randomly chosen per glass slide and the percentage of FITC-positive hemocytes, which had engulfed the labelled E. coli, was calculated after observation through a fluorescent microscope equipped with Nomarski optics (Olympus, Model BX41, Tokyo, Japan).

\section{Nodulation assay}

V-1 larvae were injected with the dsRNAs. Two days later, the larvae were further injected with formaldehyde-fixed DH5 alpha $E$. coli at a dosage of $3 \times 10^{7} \mathrm{pfu}$. Twenty-four hours after injection with $E$. coli each larva was dissected, and the number and morphology of the nodules formed were recorded.

\section{Encapsulation assay}

Two days after being treated with dsRNA, VI-0 larvae were injected with ca. 20 fluorescent latex beads (Fluoresbrite Plain Microspheres, $20 \mu \mathrm{m}$ in diameter, YG in color, Polysciences, Inc., Warrington, PA, USA). Twelve hours after the injection of beads, the larvae were dissected under a fluorescent stereoscopic microscope (Olympus, Model SZX12) and the fluorescent beads were recovered. The recovered beads were then observed using a microscope equipped with Nomarski optics, classified into three categories based on the degree of encapsulation and frequency of each of the following categories was determined: category A, beads surrounded by more than ten layers of flattened cells; category B, beads surrounded by only a few cell layers; category C, naked beads or those with only a few attached cells.

\section{RESULTS \\ cDNA cloning and sequence analyses of $\mathrm{MsNrg}$}

To obtain a full nucleotide sequence of $M s N r g$ cDNA, we started with a homologous RT-PCR approach based on the amino acid sequences of insect neuroglian proteins so far reported. Two pairs of degenerate PCR primers, one of which was used for the amplification of the region located in the $\mathrm{N}$-terminal and the other for the region in the C-terminal half, were synthesized and used for RT-PCR with oligo-(dT) primed 1st strand cDNA prepared from total hemocyte RNA. The former region (open bar in Fig. 1, about $670 \mathrm{bp}$ ) was successfully amplified while the latter region (filled bar in Fig. 1, about 660 bp) was not. We considered the possibility that strong secondary structures of $M s N r g$ mRNA might have interfered with the reverse transcription of this region. To circumvent this potential 

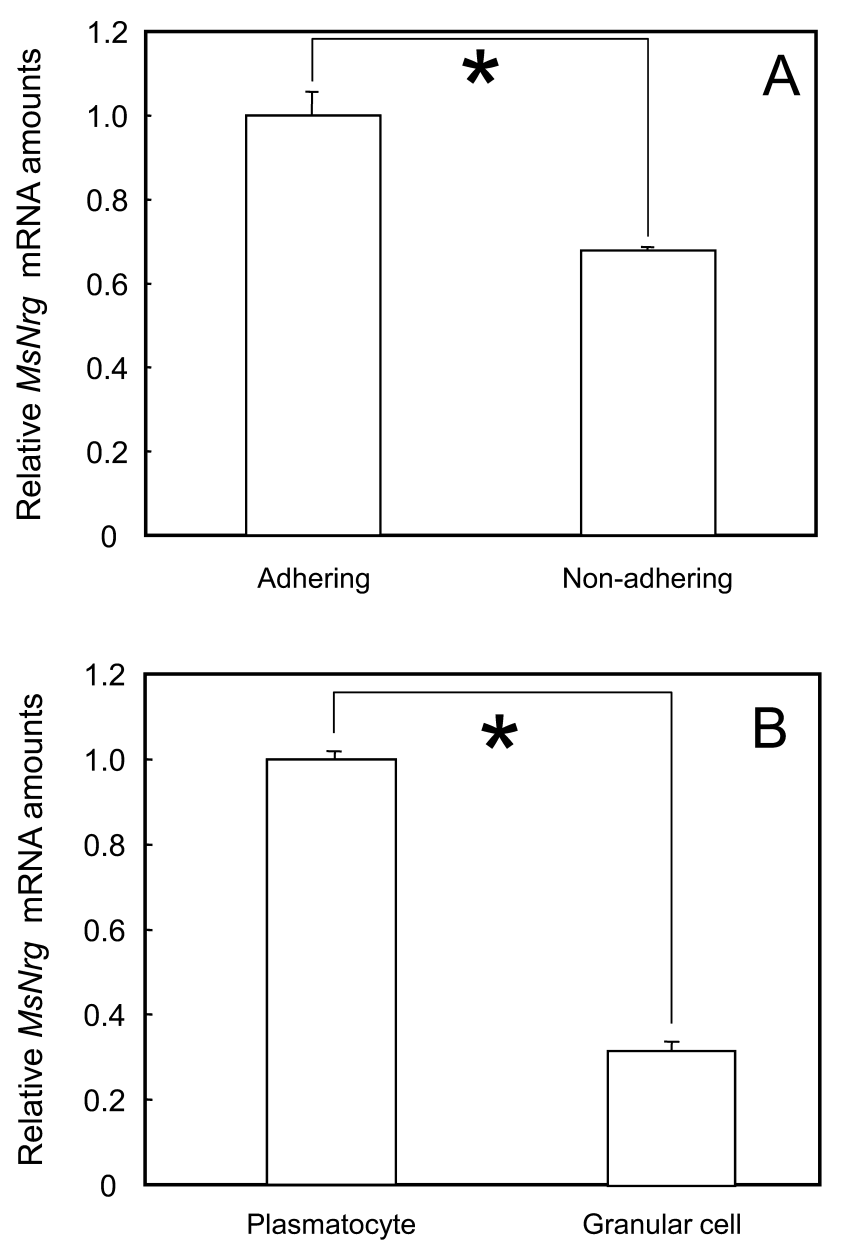

Fig. 3. MsNrg mRNA levels determined using qRT-PCR in (A) (B), (E) and (F). Distribution of MsNrg mRNA between adhering hemocytes determined using in situ hybridization in (C) and (D). (A) MsNrg mRNA levels were determined in adhering and nonadhering hemocytes. The raw values were normalized using the numbers of cells. The average value for the adhering hemocytes is set to 1.0. Vertical bars represent standard deviation (S.D.). The MsNrg mRNA is more abundant in adhering than in non-adhering hemocytes. Asterisk indicates $p<0.05$ between adhering and nonadhering hemocyte samples (two sample $t$-test), based on three replicates. (B) MsNrg mRNA distribution among two types of adhering hemocytes, plasmatocytes and granular cells. The raw values were normalized based on the number of cells. The average value for plasmatocytes is set to 1.0. The values for plasmatocytes are significantly higher than those for granular cells. Asterisk indicates $p<0.05$ between plasmatocyte and granular cell samples (two sample $t$-test), based on three replicates. (C) and (D) In situ hybridization of adhering hemocytes from sixth instar larvae. Antisense (C) or sense (D: as negative control) oligonucleotides of MsNrg labelled with DIG were used. Thin arrows and bold arrows indicate typical granular cells and spreading plasmatocytes, respectively, and scale bars represent $50 \mu \mathrm{m}$. (E) and $(F)$ Changes in the level of MsNrg mRNA in adhering hemocytes (E) and nonadhering hemocytes $(F)$ three hours after injection with either PBS (control), fluorescent latex beads or fixed $E$. coli. The raw values were normalized based on the number of cells. The average value for hemocytes of the larvae injected with PBS is set to 1.0. Vertical bars represent S.D. Based on three replicates of each category and a two sample $t$-test, there are no significant differences $(p>$ $0.05)$ (control samples versus the samples of each treatment).

problem, we carried out the reverse transcription step at a higher temperature $\left(65^{\circ} \mathrm{C}\right)$ with a thermostable enzyme, which resulted in the amplification of this region. The two
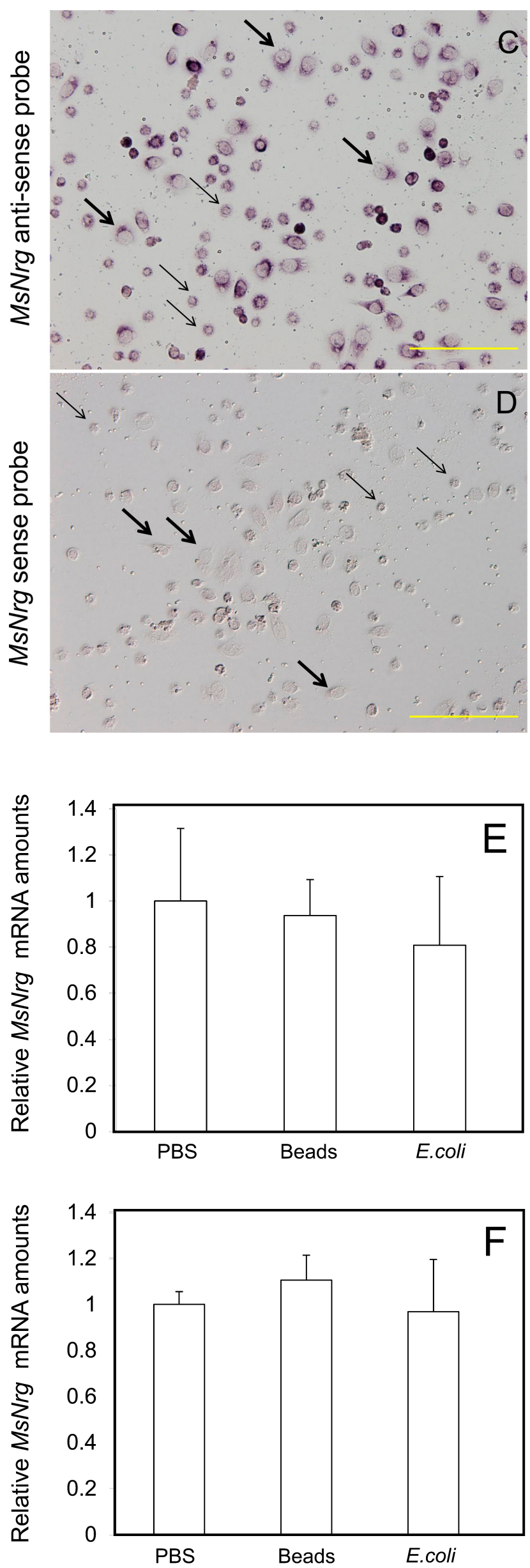
cDNA fragments were sub cloned and sequenced, revealing that these fragments encoded portions of the MsNrg polypeptide. Then, the unidentified region between the two sequences (diagonally hatched bar in Fig. 1) was amplified with a gene specific primer pair whose design was based on the sequences of the 660 and $670 \mathrm{bp}$ regions. The amplified fragment was gel-purified and sequenced directly.

The first strand cDNA used for 5' RACE was synthesized with oligo-(dT) priming under the presence of an RNA oligo bearing the 5' anchor sequence described in the Materials and Methods. This cDNA preparation was subjected to RT-PCR with the 5' anchor primer and a gene specific primer, yielding a single cDNA band with an apparent size of $1 \mathrm{kbp}$. The cDNA fragment was sequenced directly, giving the extreme 5' end sequence of $M s N r g$ cDNA (vertically hatched bar in Fig. 1).

To amplify the extreme 3 ' region (horizontally hatched bar in Fig. 1), reverse transcription was done at $65^{\circ} \mathrm{C}$ using the thermostable enzyme with the tagged oligo-(dT) primer. The following RT-PCR resulted in relatively poor amplification and banding. So, we utilized Southern hybridization to identify the positive cDNA band on a gel and selected the positive colonies after the sub cloning procedure. Then, a few independent clones were sequenced, and the consensus sequence for the extreme 3' end was determined. These respective sequences were combined, giving the full nucleotide sequence for $M s N r g$ cDNA. The whole sequence data was registered in Genbank (accession no. AB490501).

Complete cDNA and predicted amino acid sequences of MsNrg are shown in Fig. 2. The cDNA consists of 4,130 bp without its poly(A) tail. It has a 5' untranslated region (UTR) of $148 \mathrm{bp}$ and a 3' UTR of $220 \mathrm{bp}$. The 3' UTR contained a polyadenylation signal (agtaaa) $26 \mathrm{bp}$ upstream from the polyadenylation site (Beaudoing et al., 2000). The cDNA encodes an 1,254 amino acid-polypeptide with a predicted molecular mass of 140, 020.58 Da.

The predicted amino acid sequence of MsNrg was aligned with the counterparts of $M$. sexta Nrg and D. melanogaster Nrg using the CLUSTAL W program at the DNA data bank of Japan website (data not shown). Amino acid residues were highly conserved in the $M$. sexta $\mathrm{Nrg}$ and $D$. melanogaster Nrg. A cluster of hydrophobic amino acid residues was found near the C-terminus, which might include a transmembrane domain (Fig. 2, amino acid residues in bold italics). MsNrg has an 86 and $57 \%$ sequence identity with $M$. sexta $\mathrm{Nrg}$ and $D$. melanogaster $\mathrm{Nrg}$, respectively. Protein domain analysis of MsNrg using the NCBI Conserved Domain Search program server revealed that the MsNrg contains six immunoglobulin-like domains, five of which represent IGcam (immunoglobulin cell adhesion molecule domain), and five fibronectin type three (FNIII) domains, from the $\mathrm{N}$ - to $\mathrm{C}$-terminus, which are typical of neuroglian family members (data not shown).

\section{Distribution of MsNrg mRNA among hemocyte populations and immune stimuli induced changes in the amount of MsNrg mRNA}

To investigate distribution of $M s N r g$ mRNA, we determined mRNA amount in several hemocyte populations using qRT-PCR. First, hemocytes were separated into adhering (granular cells and plasmatocytes) and non-adhering cell types (spherule cells, oenocytoids and prohemocytes), and the total RNA was extracted separately. After reverse transcription, samples were subjected to qRT-PCR, and the initial level of $M s N r g$ mRNA was calculated using the 2nd derivative maximum method. Since the mRNA levels of housekeeping genes, namely RPL32 and cytoplasmic actin, differed greatly within each hemocyte category, we used cell numbers to normalize the values for $M s N r g$ mRNA. The results are shown in Fig. 3A. The $M s N r g$ expression in the adhering hemocytes was as expected but significant levels of expression were unexpectedly recorded in nonadhering hemocytes. Although the MsNrg should have a role in these non-adhering hemocytes, we did not examine this further in this study.

Distribution of $M s N r g$ mRNA among adhering hemocytes was analyzed after separation of the different cell types. Hemocyte fractions enriched in granular cells and plasmatocytes were prepared according to our previous paper (Suzuki et al., 2009). Since the procedures for isolating these hemocytes include incubation in the presence either of a growth-blocking peptide or $120 \mathrm{mM}$ EDTA, we do not exclude the possibility that these treatments may alter the expression levels of $M s N r g$. Using respective hemocyte preparations, the levels of MsNrg mRNA in the two hemocyte populations were measured (Fig. 3B). Clearly, the $M s N r g$ mRNA is much more abundant in plasmatocytes than in granular cells. Furthermore, to confirm whether MsNrg was indeed expressed in adhering hemocytes, in situ hybridization was done. Adhering hemocytes were clearly stained when the $M s N r g$ anti-sense probe (Fig. 3C) was used, but not stained when the sense probe was used (Fig. 3C). However, clear differences in the intensity of the staining of plasmatocytes and granular cells was not observed.

To confirm whether $M s N r g$ mRNA was induced by immune stimuli, intact VI-0 larvae were injected with either formaldehyde-fixed $E$. coli or fluorescent latex beads. Then, we checked the level of MsNrg mRNA in both adhering hemocytes and non-adhering hemocytes using qRTPCR (adhering hemocytes in Fig. 3E and non-adhering hemocytes in Fig. 3F). The results show that the amount of MsNrg in adhering hemocytes and non-adhering hemocytes was not changed by the immune stimuli.

\section{Involvement of MsNrg in phagocytosis}

The functions of MsNrg protein were analyzed in the context of its involvement in cellular immune defense mechanisms using RNAi. The larvae were injected with dsRNA of either EGFP or MsNrg and two days later phenotype assays were conducted. For the phagocytosis assay, FITC-labelled E. coli was injected into the larval hemo- 
Table 1. Proportion of engulfed haemocytes in control and $\mathrm{MsNrg}$ knockdown larvae.

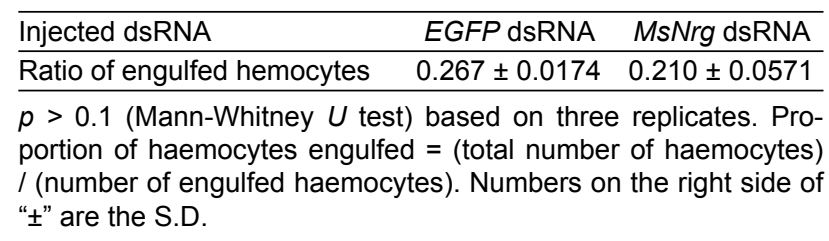

coel. The larvae were bled $3 \mathrm{~h}$ later, the hemocytes were observed with a fluorescent microscope and the percentage of hemocytes that had engulfed the labelled E. coli was calculated. Statistical analyses indicated that RNAi of $M s N r g$ did not significantly affect the phagocytotic activity more or less than the EGFP treatment (Table 1).

\section{Involvement of MsNrg in nodulation}

To assess the MsNrg involvement in nodulation, a formaldehyde-fixed $E$. coli suspension was injected into the larvae pre-injected with dsRNA of either MsNrg or EGFP. The larvae were dissected $24 \mathrm{~h}$ later, and numbers and morphology of nodules were examined. No significant difference was found in the number of nodules in the $M s N r g$ and EGFP dsRNA-treated larvae (Table 2). Nodules dissected under a stereoscopic microscope with Nomarski optics were further observed at a higher magnification. This revealed that the nodules of the EGFP dsRNA-treated larvae were morphologically indistinguishable from those from the $M s N r g$ dsRNA-treated larvae (data not shown).

\section{Involvement of MsNrg in encapsulation}

To evaluate the involvement of the MsNrg in encapsulation, fluorescent latex beads were injected into the VI-0 larvae pre-injected with dsRNA of either $M s N r g$ or EGFP as above. The larvae were bled $12 \mathrm{~h}$ later, and the beads were collected and observed using Nomarski optics. Each bead was classified as representing one of three categories according to the degrees of encapsulation (Fig. 4A-C): (A) fully encapsulated by more than ten cell layers composed of thin, flattened cells; (B) encapsulated by only a few cell layers; (C) only slightly or not encapsulated. Based on the scoring system, the beads recovered from EGFP and $\mathrm{MsNrg}$ dsRNA-treated larvae were classified and counted, and the percentages were calculated. As is evident in Fig. 4D, there was a significant difference in the degree of encapsulation recorded for hemocytes from control EGFP and the $M s N r g$ dsRNA-treated larvae. In the EGFP dsRNA-treated larvae, more than a half of the beads were in category A while in the $M s N r g$ dsRNA-treated larvae the peak shifted to category $\mathrm{B}$, indicating that the $M s N r g$ RNAi attenuated the encapsulation response. Results presented in Fig. 4D indicate that the injection of $\mathrm{MsNrg}$ dsRNA consistently reduced the amounts of corresponding mRNA species in adhering hemocytes. To assess the degree of knockdown, the qRT-PCR approach was adopted, and the qRT-PCR revealed that the $M s N r g$ knockdown in non-adhering hemocytes did not occur (data not shown). The MsNrg mRNA levels in the adhering hemocytes were determined for both EGFP and MsNrg dsRNA-treated larvae (Fig. 4E). With
Table 2. Number of nodules in control and MsNrg knockdown larvae.

Injected dsRNA EGFP dsRNA MsNrg dsRNA

Average number of nodules in larvae $28.3 \pm 2.52 \quad 33.7 \pm 7.64$

$p>0.05$ (two sample $t$-test), based on three replicates. Numbers on the right side of " \pm " are S.D.

the knockdown levels around $80 \%$ relative to the EGFP controls, encapsulation was clearly attenuated in larvae treated with $M s N r g$ dsRNA compared to those treated with EGFP dsRNA (Fig. 4D).

\section{DISCUSSON}

In the present study, the full sequence for neuroglian mRNA was obtained from cDNA derived from $M$. separata total hemocytes by combining several reverse transcription and PCR-based methods, including reverse transcription at high temperature. The $\operatorname{poly}(\mathrm{A})$ additional signal was AGTAAA, which is atypical. The poly(A) additional signal, however, has many variations and ATGAAA is the one of the variations (Beaudoing et al., 2000). Based on the predicted open reading frame, a full amino acid sequence was obtained and analyzed. MsNrg has six immunoglobulin-like domains and five FNIII domains, which is typical of neuroglian proteins. Neuroglian proteins from $M$. sexta and $D$. melanogaster are reported to bear the same domain structures as MsNrg (Bieber et al., 1989; Chen et al., 1997). The immunoglobulin-like domains have an important role in cell-cell adhesion. In Sf9 cells expressing the M. sexta Nrg transgene, cell aggregation was diminished after the addition of monoclonal antibodies that recognize immunoglobulin-like domains of $M$. sexta Nrg (Zhuang et al., 2007). These sequence analysis results imply that the M. sexta Nrg functions could be conserved in MsNrg.

MsNrg mRNA was detected in the adhering plasmatocytes and granular cells using qRT-PCR and in situ hybridization. qRT-PCR data show that MsNrg mRNA was more abundant in plasmatocytes. Of note is that the $\mathrm{MsNrg}$ mRNA was also abundant in the non-adhering hemocytes, consisting of spherule cells, oenocytoids and prohemocytes. This was an unexpected result, because the mRNA for this adhesion protein was also expressed in the population of non-adhering hemocytes. At present, we cannot exclude the possibility that the expression level of $M s M r g$ might be affected by incubation of hemocytes in culture bottles used for separating adhering and non-adhering hemocytes from total hemocytes. The result implies that $M s N r g$ could have unidentified functions in non-adhering hemocytes. Investigation of the functions of MsNrg could be a subject for future studies.

Functional assays of MsNrg in terms of its involvement in cellular immune defense were done using the RNAi procedure. As for the involvement in phagocytotic activity, no significant difference was found between the hemocytes from the $M s N r g$ dsRNA- and control EGFP dsRNA-treated larvae, suggesting that MsNrg may not be involved in phagocytosis. A few immunoglobulin superfamily members have been shown to play a role in phagocytosis in 

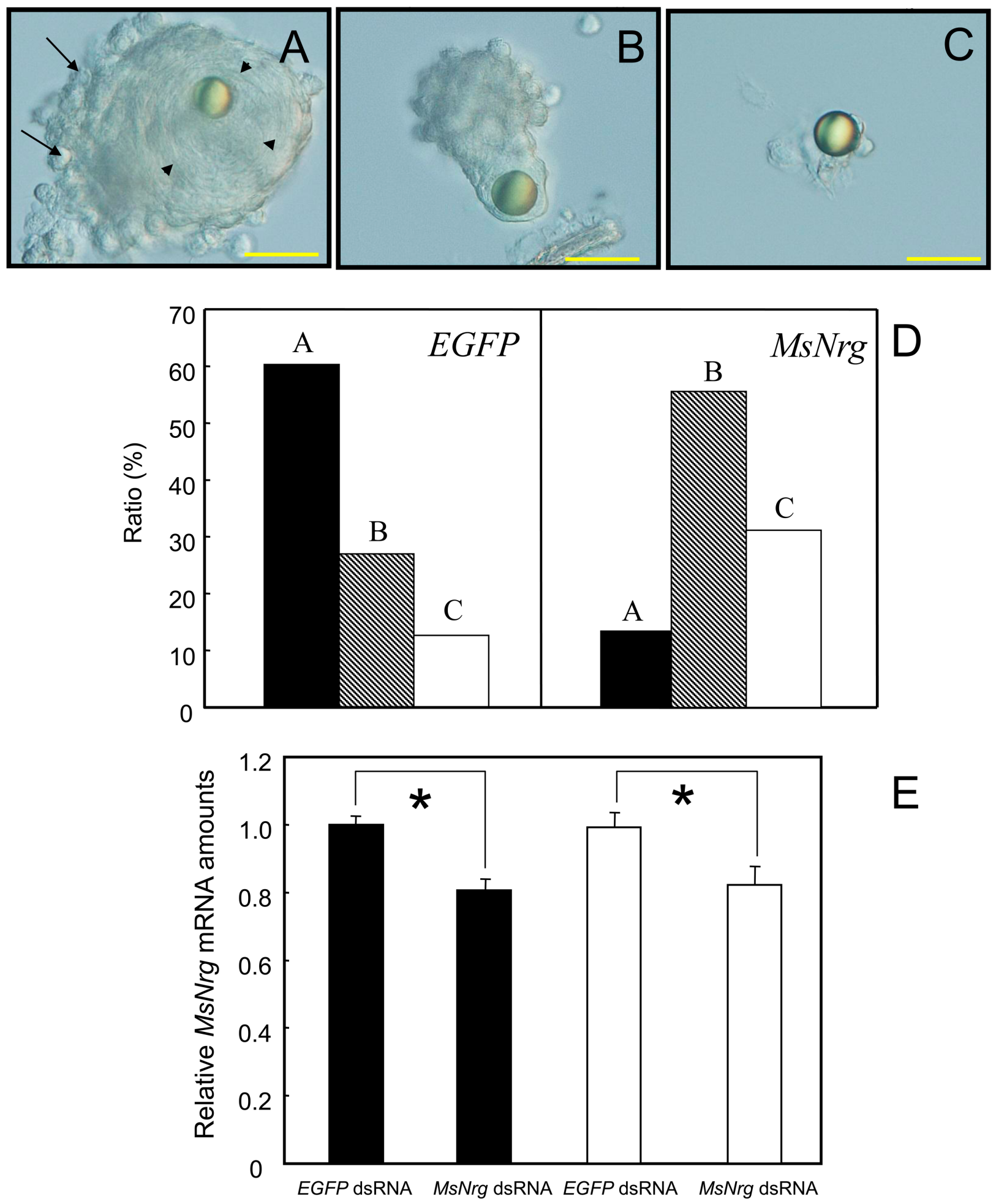

EXP-1

EXP-2

Fig. 4. Scoring the incidence of the encapsulation categories (A-C). Examples of three classes of encapsulated beads are presented. (A) The bead is fully encapsulated with more than 10 layers of thin, flattened cells (arrowheads). Cells with globular shapes form the outer layer (arrows). (B) The bead is surrounded by only a few cell layers. (C) There are either no cells or only a few cells attached to the beads. Scale bars indicate $50 \mu \mathrm{m}$. (D) Distribution of encapsulation categories (Fig. 4A-C) for beads recovered from dsRNA-treated larvae. The fluorescent latex beads were collected from the hemocoel of larvae pre-treated with dsRNA of either EGFP or MsNrg, and the degrees of encapsulation for each bead was determined according to the scoring shown in Fig. 4A-C. The percentages of the respective categories are shown. Three larvae were used for the EGFP and MsNrg RNAi, and the experiment was repeated three times. Data shown are the sum of the three independent experiments ( $p<0.01$, chi-square test). (E) Changes in levels of MsNrg mRNA in adhering hemocytes after dsRNA treatment. Larvae were treated with either EGFP or MsNrg dsRNA. Two days later, the levels of MsNrg mRNA in the adhering hemocytes were determined. The values were normalized based on the number of cells. The experiments were repeated twice, and two sets of results are presented. Sample numbers for each category are three. Asterisks indicate $p<0.05$ between control and the samples injected with MsNrg dsRNA (two sample $t$-test). The average value for EGFP in experiment-1 is set to 1.0. Vertical bars represent S.D. 
other insects. The Down syndrome cell adhesion molecule (Dscam), which belongs to the immunoglobulin superfamily, functions as a phagocytotic receptor on the surface of D. melanogaster hemocytes (Watson et al., 2005). Like neuroglian proteins, Dscam has multiple immunoglobulinlike and FN III domains. Hemolin is another member of immunoglobulin superfamily (Sun et al., 1990), and it occurs only in Lepidoptera. Eleftherianos et al. (2007) report that Manduca hemocytes in which hemolin was knocked down took up fewer $E$. coli than the control hemocytes. These other members of the immunoglobulin superfamily (Dscam, hemolin) may also be responsible for phagocytosis by $M$. separata hemocytes.

Significant difference was not found in nodulation between $M s N r g$ and EGFP dsRNA-treated larvae. The results indicate that MsNrg does not play an indispensable role in nodulation. Data in the literature suggest that cell-cell adhesion is assumed to be an important process in nodulation in which adhering hemocytes play a central role. Nodule formation includes a process of spreading and flattening of plasmatocytes (Dean et al., 2004). Faraldo et al. (2008) present a morphological study showing that nodules consist of spread and non-spread plasmatocytes and granular cells, which are surrounding injected yeast cells. Nodulation is a complex process involving several distinct molecules, such as eicosanoids, prophenoloxidase and dopadecarboxylase, in addition to adhesion molecules (Marmaras \& Lampropoulou, 2009). During the process of cell-cell adhesion in nodulation, several distinct adhesion molecules may participate. Under such circumstances, the RNAi knockdown effect on one adhesion molecule could be rescued by the redundancy provided by other molecular species. Therefore, we cannot exclude the functional involvement of MsNrg in nodulation, even though attenuation of nodulation was not observed after treatment with $M s N r g$ RNAi. The degree of $M s N r g$ knockdown was moderate after the dsRNA treatment. This moderate knockdown brought about a clear shift in phenotype for encapsulation, but not for nodulation. This may reflect the involvement of multiple adhesion molecules in the process of cell-cell adhesion during nodulation. The possibility that more effective $M s N r g$ knockdown might result in clearer changes in nodulation phenotypes also exists.

The encapsulation of injected latex beads by hemocytes was weakened by the MsNrg dsRNA treatment. The degree of attenuation is modest, which may correspond to the modest, $80 \%$ relative to the control, $M s N r g$ knockdown determined by qRT-PCR. Microscopic observation revealed that after $M s N r g$ dsRNA treatment the numbers of beads surrounded by multiple layers of thin, flattened cells decreased significantly and consistently. According to morphological features of these cells, the thin and flattened cells were probably plasmatocytes (Lavine \& Strand, 2002). In $M$. sexta, when the larvae were treated with $M$. sexta Nrg dsRNA, the numbers of fully encapsulated Sephadex beads decreased to $50 \%$ (Zhuang et al., 2007). In D. melanogaster, improper encapsulated wasp eggs were observed in D. melanogaster expressing mutant Nrg (Wil- liams, 2009). Our result and the two reports for $M$. sexta and $D$. melanogaster suggested that the cellular immunological role of neuroglian was conserved within Lepidoptera and D. melanogaster.

We show that MsNrg is involved in encapsulation of latex beads (abiotic targets), which indicates that MsNrg is involved in the cellular immune reaction against abiotic targets. Williams (2009) shows that a mutant of $\mathrm{Nrg}$ in Drosophila incompletely encapsulated parasitoid wasp eggs (biotic targets). Considering both this report and our results, MsNrg can function against both biotic and abiotic targets in at least encapsulations.

The qRT-PCR revealed an abundance of $M s N r g$ mRNA in plasmatocytes, three-times more abundant than in granular cells. When the larvae were treated with $\mathrm{MsNrg}$ dsRNA, the percentage of fully encapsulated fluorescent latex beads decreased to less than $25 \%$ of the control. Assays using qRT-PCR showed that the associated level of knockdown was only modest (Fig. 4E). This indicates that an efficient knockdown might occur in some $\mathrm{MsNrg}$ expressing subpopulations of plasmatocytes, which are involved in encapsulation. Given these results, MsNrg is likely to function as major adhesion molecule in the formation of multiple plasmatocyte layers surrounding large invading non-selves. We did these mRNA quantifications without separating adhering hemocytes into populations of granular cells and plasmatocytes, since the separation procedures require long incubation periods and relatively severe treatments (Suzuki et al., 2009), which could affect gene expression.

In this study we determined the distribution and levels of expression of $M s N r g$ mRNA mainly in hemocyte preparations consisting of two or more types of cells using qRTPCR and in situ hybridization. These results revealed the $M s N r g$ was expressed not only in adhering hemocytes but also in non-adhering hemocytes, which are not thought to be involved the cellular immune reactions. In addition, we revealed using RNAi that MsNrg was involved in the cellar immune reaction, encapsulation. Our results showed that neuroglian could have unidentified function in nonadhering hemocytes, and the cellular immunological function of neuroglian is conserved within Lepidoptera and $D$. melanogaster.

ACKNOWLEDGEMENTS. We thank T. Tanaka for his helpful technical advice. Also, we appreciate the advice of D. Kageyama, which greatly improved our manuscript. This work was supported in part by Grant-in-Aid 23658047 and 25450486, to KM from the Japan Society for the Promotion of Science.

\section{REFERENCES}

Beaudoing E., Freier S., Wyatt J.R., Claverie J.M. \& Gautheret D. 2010: Patterns of variant polyadenylation signal usage in human genes. — Genome Res. 10: 1001-1010.

Bieber A.J., Snow P.M., Hortsch M., Patel N.H., Jacobs J.R., Traquina Z.R., Schilling J. \& Goodman C.S. 1989: Drosophila neuroglian: a member of the immunoglobulin superfamily with extensive homology to the vertebrate neural adhesion molecule L1. — Cell 59: 447-460. 
Chen C.L., Lampe D.J., Robertson H.M. \& Nardi J.B. 1997: Neuroglian is expressed on cells destined to form the prothoracic glands of Manduca embryos as they segregate from surrounding cells and rearrange during morphogenesis. - Dev. Biol. 181: 1-13.

Da Silva J.B., De Albuquerque C.M., De Araujo E.C., Peixoto C.A. \& Hurd H. 2000: Immune defense mechanisms of Culex quinquefasciatus (Diptera: Culicidae) against Candida albicans infection. - J. Invertebr. Pathol. 76: 257-262.

Dean P., Richards E.H., Edwards J.P., Reynolds S.E. \& CharnLEY K. 2004: Microbial infection causes the appearance of haemocytes with extreme spreading ability in monolayers of the tobacco hornworm Manduca sexta. - Dev. Comp. Immunol. 28: 689-700.

Eleftherianos I., Gökçen F., Felföldi G., Millichap P.J., Trenczek T.E. French-Constant R.H. \& Reynolds S.E. 2007: The immunoglobulin family protein Hemolin mediates cellular immune responses to bacteria in the insect Manduca sexta. Cell Microbiol. 5: 1137-1147.

Faraldo A.C., Gregorio E.A. \& Lello E. 2008: Morphological and quantitative aspects of nodule formation in hemolymph of the blowfly Chrysomya megacephala (Fabricius, 1794). Exp. Parasitol. 118: 372-377.

Hall S.G. \& Bieber A.J. 1997: Mutations in the Drosophila neuroglian cell adhesion molecule affect motor neuron pathfinding and peripheral nervous system patterning. $-J$. Neurobiol. 32: $325-340$.

Hernandez S., Lanz H., Rodriguez M.H., Torres J.A., MartinezPalomo A. \& Tsutsumi V. 1999: Morphological and cytochemical characterization of female Anopheles albimanus (Diptera: Culicidae) hemocytes. - J. Med. Entomol. 36: 426-434.

HorTsCH M. 1996: The L1 family of neural cell adhesion molecules: Old proteins performing new tricks. - Neuron 17: 587-593.

Hortsch M., Wang Y.M., Marikar Y. \& Bieber A.J. 1995: The cytoplasmic domain of the Drosophila cell adhesion molecule neuroglian is not essential for its homophilic adhesive properties in S2 cells. - J. Biol. Chem. 270: 18809-18817.

Kato Y., Yoshida T., Miura K., Tanaka T., Nakamatsu Y. \& OCHIAI M. 2014: A novel type of hemocytes localizing melanization with high-spreading behavior in Mythimna separata. - Arch. Insect Biochem. Physiol. 86: 220-239.

LAVINE M.D. \& STRAND M.R. 2002: Insect hemocytes and their role in immunity. - Insect Biochem. Mol. Biol. 32: 1295-1309.

Lemaitre B. \& Hoffmann J. 2007: The host defense of Drosophila melanogaster. - Annu. Rev. Immunol. 47: 697-743.

Marchler-Bauer A., Bo Y., Han L., He J., Lanczycki C.J., Lu S., Chitsaz F., Derbyshire M.K., Geer R.C., Gonzales N.R. ET AL. 2017: CDD/SPARCLE: Functional classification of proteins via subfamily domain architectures. - Nucl. Acids Res. 45: D200-D203.

Marmaras V.J. \& LAmpropoulou M. 2009: Regulators and signalling in insect hemocyte immunity. - Cell Signal. 21: 186-195.
Nakamatsu Y., Kuriya K., Harvey J.A. \& Tanaka T. 2006: Influence of nutrient deficiency caused by host developmental arrest on the growth and development of a koinobiont parasitoid. J. Insect Physiol. 52: 1105-1112.

NARDI J.B. 1994: Rearrangement of epithelial cell types in an insect wing monolayer is accompanied by differential expression of a cell surface protein. - Dev. Dyn. 199: 315-325.

Nardi J.B., Pilas B., Ujhelyi E., Garsha K. \& Kanost M.R. 2003: Hematopoietic organs of Manduca sexta and hemocyte lineages. - Dev. Genes Evol. 213: 477-491.

Nardi J.B., Zhuang S., Pilas B., Bee C.M. \& Kanost M.R. 2005: Clustering of adhesion receptors following exposure of insect blood cells to foreign surfaces. - J. Insect Physiol 51: 555564.

Nardi J.B., Pilas B., Bee C.M., Zhuang S., Garsha K. \& Kanost M.R. 2006: Neuroglian-positive plasmatocytes of Manduca sexta and the initiation of hemocyte attachment to foreign surfaces. - Dev. Comp. Immunol. 30: 447-462.

Siegenthaler D., Enneking E.M., Moreno E. \& Pielage J. 2015: L1CAM/Neuroglian controls the axon-axon interactions establishing layered and lobular mushroom body architecture. $-J$. Cell Biol. 208: 1003-1018.

Sun S.C., Lindstrom I., Boman H.G., FaYe I. \& Schmidt O. 1990: Hemolin: An insect-immune protein belonging to the immunoglobulin superfamily. - Science 250: 1729-1732.

SuZuKi M. \& TANAKA T. 2007: Development of Meteorus pulchricornis and regulation of its noctuid host, Pseudaletia separata. - J. Insect Physiol. 53: 1072-1078.

SuzuKi M., MiURA K. \& TANAKA T. 2008: The virus-like particles of a braconid endoparasitoid wasp, Meteorus pulchricornis, inhibit hemocyte spreading in its noctuid host, Pseudaletia separata. - J. Insect Physiol. 54: 1015-1022.

SuzuKi M., Miura K. \& TANAKA T. 2009: Effects of the virus-like particles of a braconid endoparasitoid, Meteorus pulchricornis, on hemocytes and hematopoietic organs of its noctuid host, Pseudaletia separata. - Appl. Entomol. Zool. 44: 115-125.

Watson F.L., Puttmann-Holgado R., Thomas F., Lamar D.L., Hughes M., Kondo M., Rebel V.I. \& Schmucker D. 2005: Extensive diversity of Ig-superfamily proteins in the immune system of insects. - Science 309: 1874-1878.

Wiegand C., Levin D., Gillespie J., Willott E., Kanost M.R. \& Trenczer T. 2000: Monoclonal antibody MS13 identifies a plasmatocyte membrane protein and inhibits encapsulation and spreading reactions of Manduca sexta hemocytes. - Arch. Insect Biochem. Physiol. 45: 95-108.

Williams M.J. 2009: The Drosophila cell adhesion molecule Neuroglian regulates Lissencephaly-1 localisation in circulating immunosurveillance cells. - BMC Immunol. 10: 17, 12 pp.

Zhuang S., Kelo L., Nardi J.B. \& Kanost M.R. 2007: Neuroglian on hemocyte surfaces is involved in homophilic and heterophilic interactions of the innate immune system of Manduca sexta. - Dev. Comp. Immunol 31: 1159-1167.

Received February 7, 2018; revised and accepted March 15, 2018 Published online April 10, 2018 